Pathologe 2010 · [Suppl 2] 31:296-299

DOI 10.1007/s00292-010-1318-x

Online publiziert: 24 . Juli 2010

(c) Springer-Verlag 2010

\author{
D. Berg ${ }^{1} \cdot$ H. Bronger ${ }^{2} \cdot$ A. Walch ${ }^{3} \cdot$ H. Höfler ${ }^{1,3} \cdot$ K.-F. Becker ${ }^{1}$ \\ ${ }^{1}$ Institut für Pathologie, Technische Universität München \\ ${ }^{2}$ Frauenklinik und Poliklinik, Klinikum rechts der Isar, München \\ ${ }^{3}$ Pathologie, Helmholtz Zentrum München, Neuherberg
}

\title{
Analyse von Signalwegen in formalinfixierten Brustkrebsgeweben
}

\section{Identifizierung deregulierter Signalwege}

Über die Interaktion von Signalmolekülen und Rezeptoren werden intrazelluläre Signaltransduktionswege reguliert und in der Zelle Proliferation, Apoptose, Differenzierung und Adhäsion beeinflusst. Bei der Tumorprogression und Onkogenese spielen Veränderungen in diesen Rezeptoren (z. B. EGFR, HER2, ERa) und die von ihnen beeinflussten nachgeschalteten Signalwege eine äußerst wichtige Rolle. Derzeit sind einige Methoden zur Blockade solcher Signalketten bekannt, z. B. die Inhibition von HER2 durch Trastuzumab oder die Blockade von „Downstream-Effektormolekülen" wie MAP-Kinasen oder $\mathrm{PI}_{3} \mathrm{~K}$ [1].

Eine sehr attraktive Methode, um dynamische Prozesse in Tumorzellen und komplexe Vernetzungen ihrer Signalketten darstellen zu können, ist der Protein-Mikroarray [2-9]. Ein typischer Protein-Mikroarray besteht aus Nanolitermengen von Zell- oder Gewebelysaten, die mit einem Roboter auf einen mit Nitrozellulose beschichteten Glas-Slide aufgebracht werden. Anschließend wird das Zielprotein mit einem spezifischen Antikörper direkt oder indirekt detektiert. Die Proben werden dabei in Verdünnungsrei-

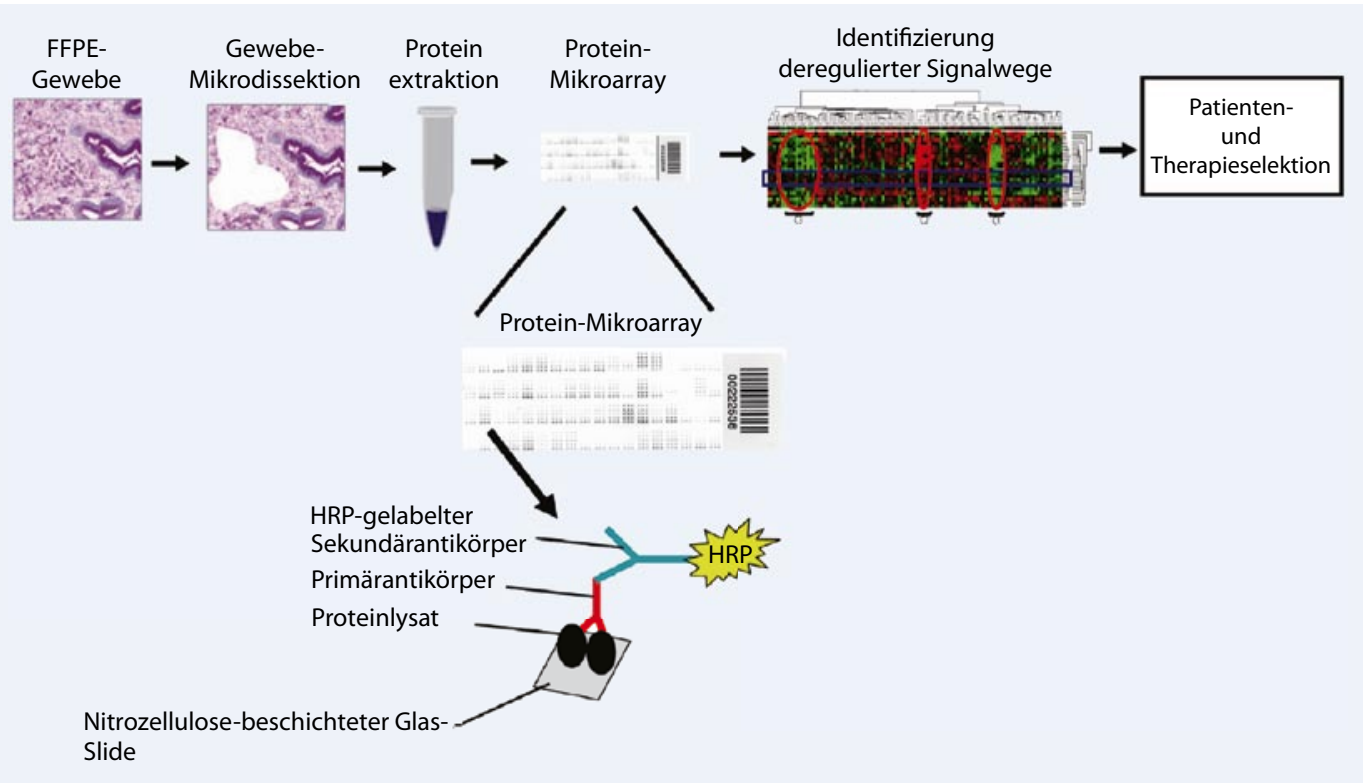

Abb. $1<$ Analyse von Signalwegen in FFPE-Geweben. Nach histologischer Analyse und Gewebe-Mikrodissektion werden Proteine aus FFPE-Gewebe extrahiert und im Westernblot und im Protein-Mikroarray analysiert. Bei dieser Methode ist im Gegensatz zu immunhistologischen Untersuchungen eine präzise Quantifizierung möglich. Dadurch können anschießend deregulierte Signalwege identifiziert werden, was die Therapie- und Patientenselektion unterstützt 
hen und Replikaten (z. B. Triplikaten) auf die Oberfläche aufgebracht. Damit wird sichergestellt, dass die Antikörper-Antigen-Reaktion immer im linearen Bereich gemessen wird. Außerdem können auf einem solchen Chip Proben vieler Tumorpatienten oder zu unterschiedlichen Zeitpunkten isolierte Proben eines Patienten untersucht und mit Referenzlysaten verglichen werden. Somit kann z. B. das Signalkaskadenprofil des Tumors eines Patienten vor, während und nach Anwendung zielgerichteter Therapien oder sich entwickelnde Resistenzen auf der Ebene der Signalproteine dargestellt werden. AuBerdem sind bei dieser Technik der Nachweis von sehr schwachen Signalen und sogar die Untersuchung von Biopsien möglich. Dies soll in Zukunft eine optimale Patienten- und Therapieselektion ermöglichen $([4,9,10,11]$, $\bullet$ Abb. 1).

Bis vor Kurzem jedoch konnte die Untersuchung von phosphorylierten Proteinen, welche den Aktivierungsstatus eines Signalwegs widerspiegeln, mittels ProteinMikroarry nur mit frischen oder tiefgefrorenen Patientenproben durchgeführt werden, da die Proteinextraktion aus FPPEGeweben nicht möglich erschien. In unserem Labor - und inzwischen auch in anderen Laboren - wurde jedoch vor Kurzem eine Technik entwickelt, mit der man vollständige, nichtdegradierte und immunreaktive Proteine aus formalinfixierten Geweben extrahieren und anschließend im Western-blot und im Protein-Mikroarray quantitativ bestimmen kann [12-19].

\section{Proteinquantifizierung}

Derzeit basiert die Patientenselektion für viele zielgerichtete Therapien auf der Ermittlung einer Überexpression des Zieproteins in formalinfixiertem Gewebe durch Immunhistochemie (IHC). Eine genaue Quantifizierung und Bestimmung des Aktivierungsstatus von Rezeptoren (z. B. HER-Rezeptoren) und nachgeschalteter Signalwegsmoleküle ist in der IHC jedoch äußerst schwierig [6]. So könnten aktivierte Rezeptoren, Rezeptorkomplexe oder deregulierte Signalwegsmoleküle erklären, warum nur ein Teil der Patientinnen auf solche Therapien ansprechen.

Pathologe 2010 - [Suppl 2] 31:296-299 DOI 10.1007/s00292-010-1318-x

(c) Springer-Verlag 2010

\section{Berg $\cdot$ H. Bronger $\cdot$ A. Walch $\cdot$ H. Höfler · K.-F. Becker Analyse von Signalwegen in formalinfixierten Brustkrebsgeweben}

\section{Zusammenfassung}

Hintergrund. Das Ziel dieser Studie ist es, Methoden zur relativen und absoluten Proteinquantifizierung in formalinfixierten und paraffineingebetteten (FFPE-) Brustkrebsgewebeproben zu entwickeln und zu optimieren, mit besonderem Schwerpunkt auf HER2vermittelte Signaltransduktion.

Material und Methoden. Mit Hilfe einer kürzlich entwickelten Methode zur Extraktion vollständiger Proteine aus FFPE-Gewebeproben testeten wir die Spezifität von mehr als 50 kommerziell erhältlichen Antikörpern im Western-blot und Protein-Mikroarray. Aufgereinigte HER-Rezeptor-Proteine wurden verwendet, um absolute Proteinkonzentrationen in FFPE-Gewebeextrakten zu bestimmen.

Ergebnisse. 23 kommerziell erhältliche phosphospezifische und nichtphosphospezi-

\section{Analysis of signalling pathways in formalin-fixed breast cancer tissues}

\section{Abstract}

Aims. The aim of our study was to develop and optimize methods for relative and absolute protein quantifications in formalin-fixed and paraffin-embedded (FFPE) tissues with special emphasis on HER mediated pathways in breast cancer.

Methods. Using a recently developed technology for extraction of full-length proteins from FFPE tissues, we evaluated $>50$ commercial antibodies for specificity using Western blots and protein microarrays. Purified HER receptor proteins were used to determine absolute protein concentrations. Results. We confirmed specificity of $23 \mathrm{com}$ mercially available phosphospecific and nonphosphospecific antibodies using Western fische Antikörper zeigten eine hohe Spezifität. Eine präzise Messung der HER2-Konzentration war möglich, indem die Signale bekannter Mengen an aufgereinigtem HER-Protein mit denen von HER-positiven Gewebeproben verglichen wurden.

Schlussfolgerung. Unsere Ergebnisse dienen als Grundlage für die Entwicklung von diagnostischen Methoden für die quantitative Analyse von deregulierten HER-Rezeptoren und nachgeschalteter Signalwegsproteine in FFPE-Gewebeproben.

\section{Schlüsselwörter}

Formalinfixiertes und paraffineingebettetes (FFPE-) Gewebe - HER2 - Protein-Mikroarray . Brustkrebs · Signaltransduktion blots with protein extracts from cell lines and tissue extracts from breast cancer patients. Spiking known amounts of purified HER receptor proteins in HER receptor negative tissue extracts allowed us to precisely measure abundances of HER-receptors.

Conclusions. Our results will provide a basis for the development of diagnostic techniques for the quantitative analysis of deregulated HER receptors and downstream signalling proteins in typical clinical tissues.

\section{Keywords}

Formalin-fixed and paraffin-embedded (FFPE) tissues - HER2 - Protein microarray . Breast cancer $\cdot$ Signal transduction 


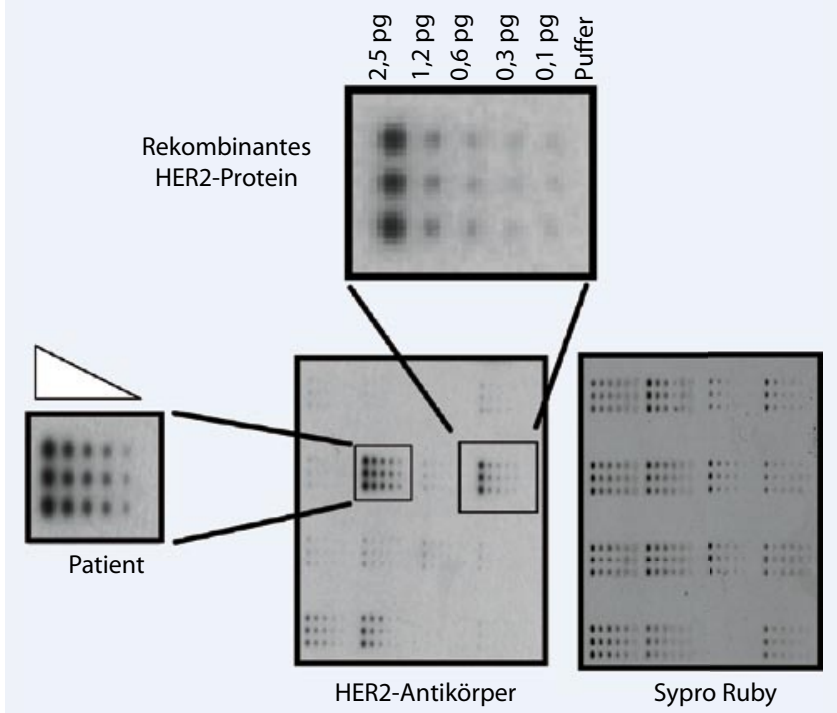

a

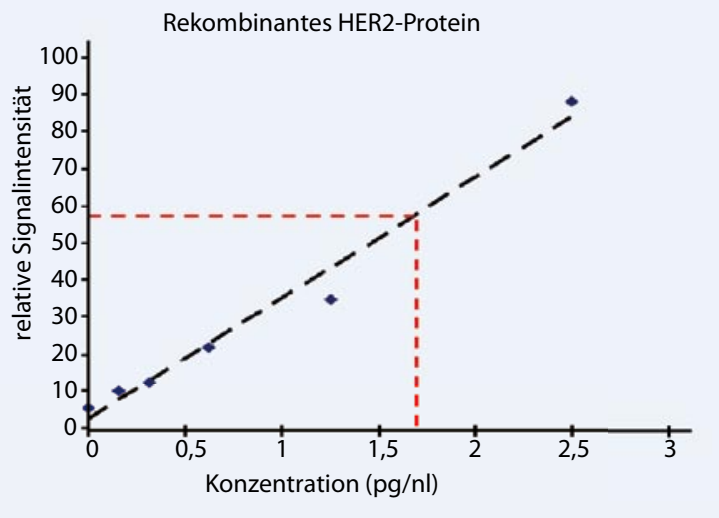

Abb. $2<$ Proteinquantifizierung durch Protein-Mikroarray. a Ein aufgereinigtes rekombinantes HER2Protein wird in einer Verdünnungsreihe zusammen mit den Patientenproben als Proteinreferenz auf einen mit Nitrozellulose beschichteten Glasobjektträger gespottet und mit einem HER2spezifischen Antikörper entwickelt. Ein mit Sypro-Ruby detektierter Array soll als Gesamtproteinreferenz dienen. $\mathbf{b}$ Es wurde eine SignalintensitätsKonzentrations-Kurve erstellt. Da die Proteinkonzentration des rekombinanten HER2Proteins bekannt ist, kann die unbekannte HER2-Konzentration in der Patientenprobe anhand der HER2-Standardkurve bestimmt werden

\section{Herausforderungen bei der Array-Analyse} akte Quantifizierung der HER2-Proteinexpression mittels Protein-Mikroarray erreicht, indem wir ein rekombinantes HER2-Protein bekannter Konzentration zusammen mit den Patientenproben in einer Verdünnungsreihe auf den Objektträger aufgebracht haben. Das HER2-Protein wurde in HER2-negativen Patientenproben verdünnt, um potenzielle Einflüsse von komplexen Proteinmischungen auf die Signalintensität zu berücksichtigen. Anschließend konnte anhand der Signalintensitäts-Konzentrations-Kurve die Proteinkonzentration in den Patientenproben ermittelt werden.

Da es sich hierbei um ein extraktbasiertes und quantitatives Verfahren handelt, ist eine Fehlinterpretation des Ergebnisses - anders als bei der IHC - ausgeschlossen. Somit ermöglicht diese Methode eine äußerst genaue und objektive Patientenselektion ([20], $\bullet$ Abb. 2).
Ein großer limitierender Faktor für eine erfolgreiche Anwendung der Protein-Mikroarray-Technik besteht in der Verfügbarkeit spezifischer Antikörper. Bei unseren eigenen Analysen werden nur Antikörper verwendet, die im Western-blot von FFPE-Lysaten ein klares Ergebnis liefern: nämlich eine spezifische Bande beim erwarteten Molekulargewicht des untersuchten Proteins (• Abb. 3). Bei mehr als 20 verschiedenen phosphospezifischen und nichtphosphospezifischen Antikörpern konnten wir eine hohe Spezifität feststellen. Eine andere Herausforderung bei der Array-Analyse ist die Variabilität und Vergleichbarkeit der Signalintensitäten zwischen verschiedenen Arrays, die mit demselben Antikörper detektiert wurden. Durch Referenzstandards, die zusammen mit den Patientenproben auf jeden Slide aufgebracht werden, lässt sich die Gesamtproteinmenge zwischen verschiedenen Arrays hervorragend vergleichen [20].

\section{Ausblick}

Obwohl die Rolle von Proteinen als wichtige Krankheitsmarker immer mehr an Bedeutung gewinnt, ist ihre schnelle quantitative Analyse in der Routine immer noch nicht möglich. Unsere Ergebnisse sollen als Basis für die Entwicklung von Methoden zur quantitativen Bestimmung von deregulierten Rezeptoren und nachgeschalteten Signalwegen in typisch klinischem Gewebe dienen.

\section{Korrespondenzadresse \\ Prof. Dr. K.-F. Becker}

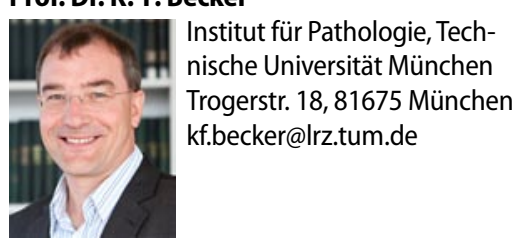

Danksagung. Unsere Arbeiten werden durch das BMBF (Förderkennzeichen Nr. 01GR0805; , FFPE-Gewebe") gefördert. Die Autoren danken Herrn Kai Tran, Frau Kerstin Schragner, Frau Christa Schott, Frau Claudia Wolff, Frau Bilge Ergin und Frau Katharina Malinowsky für ihre Unterstützung.

Interessenkonflikt. Der korrespondierende Autor weist auf folgende Beziehung hin: Herr Prof. Becker ist als Erfinder in einem Patent genannt, das die Extraktion aus FFPE-Gewebe beschreibt.

\section{Literatur}

1. Hynes NE, Lane HA (2005) ERBB receptors and cancer: the complexity of targeted inhibitors. Nat Rev Cancer 5(5):341-354

2. Becker KF, Metzger V, Hipp S et al (2006) Clinical proteomics: new trends for protein microarrays. Curr Med Chem 13(15):1831-1837

3. Calvo KR, Liotta LA, Petricoin EF (2005) Clinical proteomics: from biomarker discovery and cell signaling profiles to individualized personal therapy. Biosci Rep 25(1-2):107-125

4. Liotta LA, Espina V, Mehta Al et al (2003) Protein microarrays: meeting analytical challenges for clinical applications. Cancer Cell 3(4):317-325

5. Nishizuka S, Charboneau L, Young L et al (2003) Proteomic profiling of the $\mathrm{NCl}-60$ cancer cell lines using new high-density reverse-phase lysate microarrays. Proc Natl Acad Sci U S A 100(24):14229-14234

6. Paweletz CP, Charboneau L, Bichsel VE et al (2001) Reverse phase protein microarrays which capture disease progression show activation of pro-survival pathways at the cancer invasion front. Oncogene 20(16):1981-1989 
Abb. $3>$ Antikörpervalidierung. Die Spezifität verschiedener Antikörper wurde im Western-blot getestet. Es wurden Proteinlysate aus formalinfixierten Brustkrebsgewebeproben verwendet. Extrakte von HER2-, PTEN-, P-PTEN-, EGFR-, P-EGFR-, ERa- und PGR-positiven Geweben zeigten eine spezifische Bande beim entsprechenden Molekulargewicht. Für diese Moleküle negative Extrakte zeigten kein Signal. $\beta$-Aktin wurde als Ladekontrolle ver-

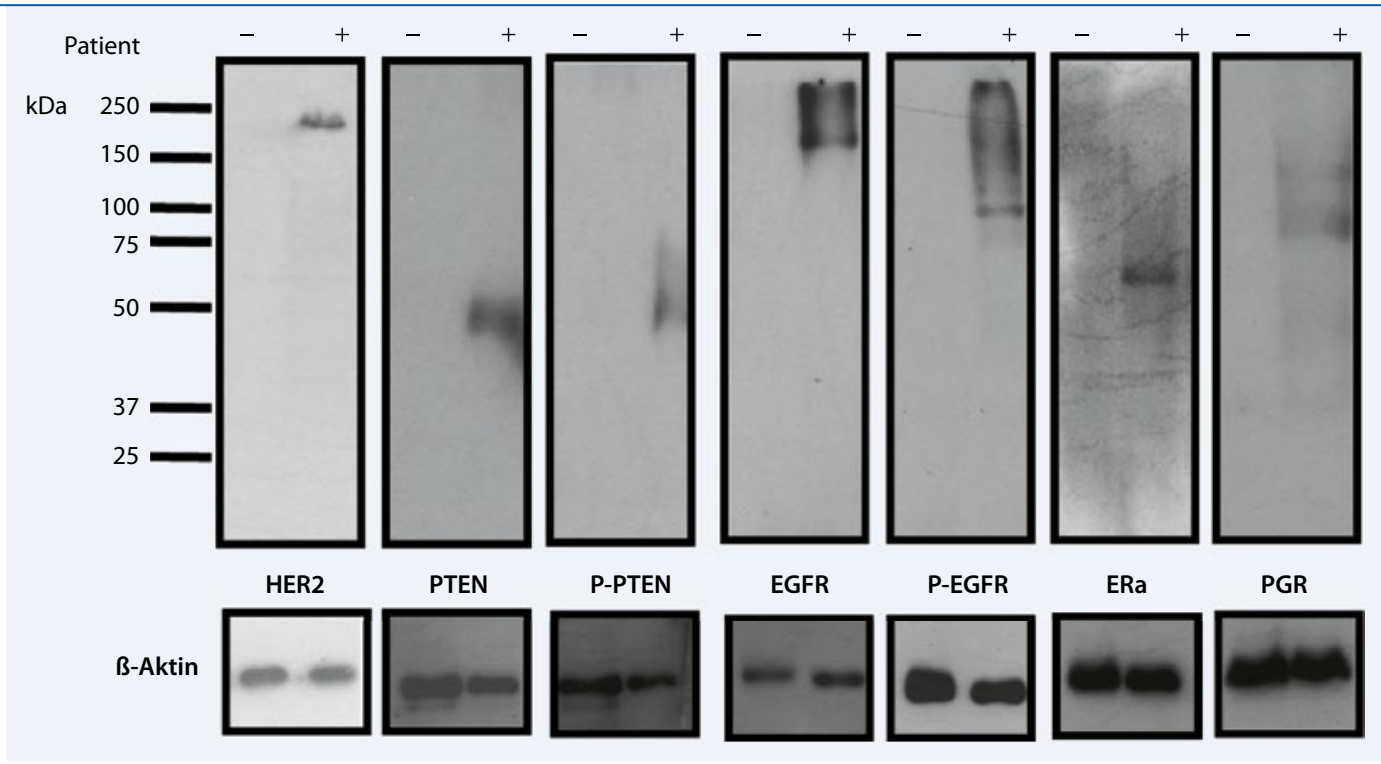

wendet

7. Petricoin EF 3rd, Bichsel VE, Calvert VS et al (2005) Mapping molecular networks using proteomics: a vision for patient-tailored combination therapy. J Clin Oncol 23(15):3614-3621

8. Sheehan KM, Calvert VS, Kay EW et al (2005) Use of reverse phase protein microarrays and reference standard development for molecular network analysis of metastatic ovarian carcinoma. Mol Cell Proteomics 4(4):346-355

9. Wulfkuhle JD, Edmiston KH, Liotta LA et al (2006) Technology insight: pharmacoproteomics for cancer-promises of patient-tailored medicine using protein microarrays. Nat Clin Pract Oncol 3(5):256268

10. Gulmann C, Sheehan KM, Kay EW et al (2006) Array-based proteomics: mapping of protein circuitries for diagnostics, prognostics and therapy guidance in cancer. J Pathol 208(5):595-606

11. Tibes R, Qiu Y, Lu Y et al (2006) Reverse phase protein array: validation of a novel proteomic technology and utility for analysis of primary leukemia specimens and hematopoietic stem cells. Mol Cancer Ther 5(10):2512-2521

12. Becker KF, Schott C, Becker I, Höfler H (2008) Guided protein extraction from formalin-fixed tissues for quantitative multiplex analysis avoids detrimental effects of histological stains. Proteomics Clin Appl 2:737-743

13. Addis MF, Tanca A, Pagnozzi D et al (2009) Generation of high-quality protein extracts from formalin-fixed, paraffin-embedded tissues. Proteomics 9(15):3815-3823

14. Becker KF, Mack H, Schott C et al (2008) Extraction of phosphorylated proteins from formalin-fixed cancer cells and tissues. TOPATJ 2:44-52

15. Chu WS, Liang Q, Liu J et al (2005) A nondestructive molecule extraction method allowing morphological and molecular analyses using a single tissue section. Lab Invest 85(11):1416-1428

16. Chung JY, Lee SJ, Kris Y et al (2008) A well-based reverse-phase protein array applicable to extracts from formalin-fixed paraffin-embedded tissue. Proteomics Clin Appl 2:1539-1547

17. Ikeda K, Monden T, Kanoh T et al (1998) Extraction and analysis of diagnostically useful proteins from formalin-fixed, paraffin-embedded tissue sections. J Histochem Cytochem 46(3):397-403
18. Nirmalan NJ, Harnden P, Selby PJ et al (2009) Development and validation of a novel protein extraction methodology for quantitation of protein expression in formalin-fixed paraffin-embedded tissues using western blotting. J Pathol 217(4):497506

19. Shi SR, Liu C, Balgley BM et al (2006) Protein extraction from formalin-fixed, paraffin-embedded tissue sections: quality evaluation by mass spectrometry. J Histochem Cytochem 54(6):739-743

20. Berg D, Hipp S, Malinowsky K et al (2010) Molecular profiling of signalling pathways in formalinfixed and paraffin-embedded cancer tissues. Eur J Cancer 46(1):47-55 